\title{
A phenomenological 10-dimension space-time model
}

\author{
Richard Bonneville \\ Centre National d’Etudes Spatiales (CNES), 2 Place Maurice Quentin, Paris, France; richard.bonneville@cnes.fr
}

Received 2 December 2013; revised 2 January 2014; accepted 9 January 2014

Copyright (c) 2014 Richard Bonneville. This is an open access article distributed under the Creative Commons Attribution License, which permits unrestricted use, distribution, and reproduction in any medium, provided the original work is properly cited. In accordance of the Creative Commons Attribution License all Copyrights @ 2014 are reserved for SCIRP and the owner of the intellectual property Richard Bonneville. All Copyright (C) 2014 are guarded by law and by SCIRP as a guardian.

\section{ABSTRACT}

The possibility of a description of the fundamental interactions of physics, including gravitation, based upon the assumption of 6 real extra dimensions is presented. The usual 4-dimension space-time, a curved surface with the Lorentz group as local symmetry, is embedded in a larger flat 10-dimension space. Through a fundamental assumption about the geometry of the orthogonal 6-d space in every point of the 4-d surface, there are two possibilities for classifying the physical states, corresponding to two types of particles: 1) hadrons, experiencing a gauge field associated to a real symmetry group $\mathrm{G}_{\mathrm{H}}(6)$, isomorphous to $\mathrm{SU}(3)$, which is identified with the strong interaction, and 2) leptons experiencing another gauge field associated with a real symmetry group $G_{L}(6)$, isomorphous to $S U(2) \times U(1)$ but different from the usual electroweak coupling. In addition, both hadrons and leptons are subject to weak and electromagnetic interactions plus a scalar BEH-like coupling, with the respective real symmetries SO(3), SO(2), $\mathrm{SO}(1)$, isomorphous to $\mathrm{SU}(2), \mathrm{U}(1), \mathrm{I}(1)$. This description can be extended so as to include gravitation; postulating a minimal Lagrangian in the full 10-d space, the equations of motion are derived. They imply the existence of a set of additional vector-type fields which do not act the same way upon hadrons and leptons, thus inducing a violation of the equivalence principle.

\section{KEYWORDS}

\section{Extra-Dimensions; Phenomenology}

\section{INTRODUCTION}

Astrophysics and particle physics tend to join together through various problems linked to the unification of the fundamental interactions of nature and the specificity of gravity with respect to the other interactions, with the ultimate goal to elaborate a new physics beyond General Relativity and the Standard Model of particle physics. The Standard Model which unifies the weak/electromagnetic/strong interactions in a single frame is based upon the common character of those interactions, a quantum field theory description of gauge symmetries within the 4-dimension space-time of special relativity. Gravitation is independently described by General Relativity, a metric theory connecting the geometry of space-time with the density of impulsion-energy. Nuclei, atoms and molecules are described by quantum theory, whereas the universe at large scale is described by General Relativity. Now in spite of its successes, General Relativity cannot be the ultimate description of gravity as it exhibits an embarrassing singularity at $\mathrm{t}=0$ and does not take into account the quantum effects, predominant in the primordial universe and at very small scales; moreover the significance of the cosmological constant $\Lambda$ is unclear. On the other hand the Standard Model accounts for the bestiary of fields and particles of ordinary matter but cannot be extended so as to include gravitation which cannot be handled through the renormalization procedures of quantum field theory. The minimal coupling between gravitation and particle physics is made by replacing, whenever needed, the pseudo Euclidean Minkowski metrics $\boldsymbol{\eta}_{u v}$ of special relativity by the $\boldsymbol{g}_{\mu v}$ metrics which expresses the curvature of space-time by its material content.

Extra dimensions in addition to the usual 4 dimensions of space-time are a common ingredient of the theoretical attempts aiming at combining gravitation with the other interactions [1-5]. Historically, the Kaluza-Klein approach in the 1920's assumes a $5^{\text {th }}$ dimension, which is enough to account for the tensor field of gravitation and for the vector field of electromagnetism. In addition, it predicts an extra scalar field and it is a common feature of unification theories to predict the existence of additional fields 
and particles. However, the later evidence of weak and strong interactions has shown that the original KaluzaKlein model was not sufficient for a comprehensive description of the fundamental interactions. The idea of introducing additional dimensions in order to account for all the known fundamental interactions including gravitation has flourished from the 1980's, e.g. with the string theories which require at least 10 dimensions to be consistent [6].

According to certain models, the size of those extra dimensions is very small, of the order of Planck's length $\left(10^{-35} \mathrm{~m}\right)$. The common image is that of a plain seen from the top of a hill: if the hill is high enough, the height of the grass is not perceived by the eye and the plain seems to be a 2-dimension domain. The extra dimensions are supposed to be compactified and wrapped so that they are hidden to us. According to other works, they could be much larger, up to $0.1 \mathrm{~mm}$ [7]. However, it is not sure that the question of the size of those dimensions is really meaningful. We experience space and time with our senses or through our instruments but the way we perceive space and time is quite different. For instance, the smallest distance we can perceive with our eyes is of the order of $0.1 \mathrm{~mm}$, and the smallest time interval we can feel is about $0.04 \mathrm{~s}$ (the interval between two images in a movie). If we convert those $0.04 \mathrm{~s}$ into length $(\mathrm{x}=\mathrm{ct})$ we get $12,000 \mathrm{~km}, 11$ orders of magnitude larger than $0.1 \mathrm{~mm}$. In the same manner as space and time are differently perceived and cannot be compared via that this type of conversion, the 6 extra dimensions might be of another nature than usual space or time; nevertheless we actually feel those extra dimensions in as much as "the fundamental interactions of particle physics" can be interpreted as the manifestations in our usual world of the geometry of the extra dimensions. If this is correct, we suggest that the connection between those 6 extra dimensions and the 3 usual dimensions of space involves a new physical constant, like the speed of light connecting space and time, which may be different from the gravitation constant which appears in Planck's length.

The goal of this paper, essentially a phenomenological approach, is to show the possibility, through a relatively simple mathematical apparatus, that a few basic assumptions on the geometrical properties of a 10-dimension space-time allow deriving the main features of the fundamental interactions: types and symmetries of the particles (hadrons and leptons), types and symmetries of the gauge fields. Moreover, this description, combined with gravitation and with the postulate of a minimal Lagrangian, predicts the existence of additional fields resulting from the coupling of gravitation with the other force fields; those hypothetical extra fields do not act the same way upon hadrons and leptons, thus inducing a violation of the equivalence principle.

\section{THE 10-DIMENSION SPACE-TIME}

The experimental confirmations of General Relativity lead us to acknowledge that, at least at a macroscopic scale, the geometry of space-time is that of a 4-dimension curved surface $\boldsymbol{\Sigma}(4)$ locally invariant under the Lorentz group $\boldsymbol{\Lambda}(4)$. On another hand a curved surface of dimension $\mathrm{d}$ can be embedded inside a flat space with $d(d+1) / 2$ dimensions; hence $\Sigma(4)$ can be embedded inside a flat space with 10 dimensions. We will hereafter make the basic assumption that our physical universe is a flat (pseudo) Euclidian space with 10 dimensions $\boldsymbol{E}(10)$, invariant under some symmetry group $\mathcal{G}(10)$.

Let $\boldsymbol{E}_{\|}(4)_{M}$ be the tangent space to $\boldsymbol{\Sigma}(4)$ in any point $\mathrm{M}$ of $\boldsymbol{\Sigma}(4)$, and $\boldsymbol{E}_{\perp}(6)_{M}$ be the orthogonal space to $\Sigma(4)$ in M. $\Sigma(4)$ is locally invariant under $\boldsymbol{\Lambda}(4)$, i.e. $\boldsymbol{E}_{\|}(4)_{M}$ is invariant under $\boldsymbol{\Lambda}(4) . \boldsymbol{E}_{\|}(4)_{M}$ is a 4dimension flat space in which one can define a system of 4 (pseudo) orthonormal coordinates $\left\{x^{\mu}\right\}$ with $\mu$ ranging from 0 to 3, i.e. one time coordinate (ct) and 3 space coordinates $(x, y, z)$. Given such a local reference frame for $\boldsymbol{E}_{\|}(4)_{M}$, one can find an infinity of other equivalent reference frames by applying to $\{c t, x, y, z\}$ any combination of rotations and Lorentz transformations while conserving the invariance of the pseudo Euclidian norm $\boldsymbol{\eta}_{\mu \nu} x^{\mu} x^{v}=-c^{2} t^{2}+x^{2}+y^{2}+z^{2}$ [8]. The effect of the local curvature of the surface can be interpreted on $\Sigma(4)$ as resulting from a force field which is identified with gravitation.

$\boldsymbol{E}_{\perp}(6)_{M}$ is a 6-dimension space. A priori we do not know anything about the metrics of this space but we will hereafter assume that $\boldsymbol{E}_{\perp}(6)_{M}$ is a flat space invariant under a symmetry group which conserves the true Euclidian norm $\sum_{i, j=1}^{6} \boldsymbol{\delta}_{i j} \xi^{i} \xi^{j}=\sum_{i=1}^{6}\left(\xi^{i}\right)^{2}$ where $\left\{\xi^{i}\right\}$ denotes a set of 6 orthonormal coordinates, i.e. it is the orthogonal group $\boldsymbol{O}(6)$ or one of its subgroups. We restrict ourselves to the special orthogonal group $\boldsymbol{S O}(6)$ as we will see later on that the transformation $\xi^{i} \rightarrow-\xi^{i}$ with $i$ ranging from 1 to 6 , equivalent in the "internal" space $\boldsymbol{E}_{\perp}(6)_{M}$ to what parity and time reversal represent in the "orbital space" $\boldsymbol{E}_{\|}(4)_{M}$, expresses the charge conjugation.

The uncertainty principle implies that the physical states are not restricted to $\Sigma(4)$ but they have an extension in the other dimensions. Thus in any point $\mathrm{M}$ of $\Sigma(4)$ there are 4 "orbital" degrees of freedom along $\boldsymbol{\Sigma}(4)$ and 6 "internal" degrees of freedom in the transverse directions.

In the following section, gravitation will be assumed to be decoupled from the other interactions; it will be introduced in a later section. 


\section{HADRONS AND LEPTONS}

\subsection{Fundamental Assumption}

We will henceforth consider the subgroups of $\boldsymbol{S O}(6)$. In the base $\left\{\xi^{i}\right\}$ any infinitesimal transformation of $\boldsymbol{S O}(6)$ can be written as [9]

$$
\boldsymbol{T}_{6}=\boldsymbol{I}(6)+\boldsymbol{M} \text { with } \boldsymbol{M}=\left(\begin{array}{cc}
\boldsymbol{A}^{\prime} & \boldsymbol{B} \\
-\tilde{B} & \boldsymbol{A}^{\prime \prime}
\end{array}\right)
$$

$\boldsymbol{M}$ is a fully antisymmetric real $6 \times 6$ matrix, $\boldsymbol{A}^{\prime}$ and $\boldsymbol{A}^{\prime \prime}$ are two antisymmetric $3 \times 3$ matrices, $\boldsymbol{B}$ is a $3 \times 3$ matrix and $\tilde{\boldsymbol{B}}$ denotes the transposed matrix of $\boldsymbol{B}$; $\boldsymbol{S O}(6)$ has got 15 infinitesimal generators.

$\boldsymbol{B}$ can be written as $\boldsymbol{S}+\boldsymbol{A}+\boldsymbol{Q}$, where $\boldsymbol{S}$ is a scalar times the 3-dimension identity $\boldsymbol{I}(3), \boldsymbol{A}$ is a $3 \times 3$ antisymmetric matrix and $\boldsymbol{Q}$ a null-trace $3 \times 3$ symmetric matrix. $\boldsymbol{M}$ can then be expressed as the sum of 3 matrices

$$
\begin{aligned}
& M= \\
& \left(\begin{array}{cc}
\boldsymbol{A}_{0} & \boldsymbol{S} \\
-\boldsymbol{S} & \boldsymbol{A}_{0}
\end{array}\right)+\left(\begin{array}{cc}
\boldsymbol{A}_{2} & \boldsymbol{Q} \\
-\boldsymbol{Q} & \boldsymbol{A}_{2}
\end{array}\right)+\left(\begin{array}{cc}
\boldsymbol{A}^{\prime}-\boldsymbol{A}_{0}-\boldsymbol{A}_{2} & \boldsymbol{A} \\
\boldsymbol{A} & \boldsymbol{A}^{\prime \prime}-\boldsymbol{A}_{0}-\boldsymbol{A}_{2}
\end{array}\right)
\end{aligned}
$$

It can be checked from their commutation relations that the matrices $\left(\begin{array}{cc}\boldsymbol{A}_{0} & \boldsymbol{S} \\ -\boldsymbol{S} & \boldsymbol{A}_{0}\end{array}\right)$ and $\left(\begin{array}{cc}\boldsymbol{A}_{2} & \boldsymbol{Q} \\ -\boldsymbol{Q} & \boldsymbol{A}_{2}\end{array}\right)$ generate 2 subgroups of $\boldsymbol{S O}(6) \boldsymbol{G}_{L}(6)$ and $\boldsymbol{G}_{H}(6)$ with respectively 4 and 8 infinitesimal generators. Those 2 subgroups have rather similar structures since

$$
\left(\begin{array}{cc}
A_{0} & S \\
-S & A_{0}
\end{array}\right)=A_{0} \otimes I(2)+S \otimes J
$$

and

$$
\left(\begin{array}{cc}
\boldsymbol{A}_{2} & \boldsymbol{Q} \\
-\boldsymbol{Q} & \boldsymbol{A}_{2}
\end{array}\right)=\boldsymbol{A}_{2} \otimes \boldsymbol{I}(2)+\boldsymbol{Q} \otimes \boldsymbol{J}
$$

where $\boldsymbol{J}=\left(\begin{array}{cc}0 & 1 \\ -1 & 0\end{array}\right)$ is the single generator of the special orthogonal group $\boldsymbol{S O}(2)$.

From the commutation relations of their infinitesimal generatorsit can be checked that $\boldsymbol{G}_{L}(6)$ is isomorphous to $\boldsymbol{S U}(2) \times \boldsymbol{U}(1)$ and that $\boldsymbol{G}_{H}(6)$ is isomorphous to $\boldsymbol{S U}(3)$.We also note that $\boldsymbol{G}_{L}(6)$ and $\boldsymbol{G}_{H}(6)$ are 2 invariant, or distinguished, subgroups of $\boldsymbol{S O}(6)$. Those 2 groups have in common $\boldsymbol{S O}(3) \otimes \boldsymbol{I}(2)$ as a maximal subgroup, where $\boldsymbol{I}(2)$ denotes the 2-dimension identity.

$$
\text { The matrices }\left(\begin{array}{cc}
\boldsymbol{A}^{\prime}-\boldsymbol{A}_{0}-\boldsymbol{A}_{2} & \boldsymbol{A} \\
\mathbf{A} & \boldsymbol{A}^{\prime \prime}-\boldsymbol{A}_{0}-\boldsymbol{A}_{2}
\end{array}\right) \text { do not }
$$

generate an invariant subgroup of $\boldsymbol{S O}(6)$ since

$$
\begin{aligned}
& \left(\begin{array}{cc}
\boldsymbol{A}^{\prime}-\boldsymbol{A}_{0}-\boldsymbol{A}_{2} & \boldsymbol{A} \\
\boldsymbol{A} & \boldsymbol{A}^{\prime \prime}-\boldsymbol{A}_{0}-\boldsymbol{A}_{2}
\end{array}\right) \\
& =\boldsymbol{A} \otimes \sigma_{\mathrm{x}}+\left(\frac{\boldsymbol{A}^{\prime}+\boldsymbol{A}^{\prime \prime}}{2}-\boldsymbol{A}_{0}-\boldsymbol{A}_{2}\right) \otimes \boldsymbol{I}(2)+\left(\frac{\boldsymbol{A}^{\prime}-\boldsymbol{A}^{\prime \prime}}{2}\right) \otimes \sigma_{\mathrm{z}}
\end{aligned}
$$

where the $\sigma_{i}$ are the Pauli matrices (the pair $\sigma_{x}, \sigma_{z}$ does not generate a group).

We here make the following fundamental assumption: only the invariant subgroups of $\boldsymbol{S O}(6)$ can be symmetry groups for the extension of the physical states in $\boldsymbol{E}_{\perp}(6)_{M}$. In other words, $\boldsymbol{M}$ shall be of the form

$$
\left(\begin{array}{cc}
\boldsymbol{A}_{0}+\boldsymbol{A}_{2} & \boldsymbol{S}+\boldsymbol{Q} \\
-\boldsymbol{S}-\boldsymbol{Q} & \boldsymbol{A}_{0}+\boldsymbol{A}_{2}
\end{array}\right)
$$

As a consequence only the pair of subgoups $\boldsymbol{G}_{H}(6)$ and $\boldsymbol{G}_{L}(6)$ is relevant to characterize the physical states, which accordingly can be classified after the irreducible representations of $\boldsymbol{G}_{H}(6)$ and $\boldsymbol{G}_{L}(6)$, the same as those of $\boldsymbol{S U}(3)$ and $\boldsymbol{S U}(2) \times \boldsymbol{U}(1)$ respectively. We can thus evidence two types of "internal" physical states, each type being characterized by a symmetry group: the "hadronic states" experiencing a $\boldsymbol{G}_{L}(6)$ symmetry, and the "leptonic states" experiencing a $\boldsymbol{G}_{H}(6)$-symmetry. Those two local symmetries can be interpreted through the existence of two gauge fields respectively acting upon the hadrons for $\boldsymbol{G}_{H}(6)$ and upon the leptons for $\boldsymbol{G}_{L}(6)$.

\subsection{Hadrons}

Agauge field identified with the strong interaction can be associated to the symmetry group $\boldsymbol{G}_{H}(6) . \boldsymbol{G}_{H}(6)$ like $\boldsymbol{S U}(3)$ has 8 infinitesimal generators which subtend a 8-dimension representation of $\boldsymbol{G}_{H}(6)$. Any given irreducible representation of $\boldsymbol{G}_{H}(6)$ is characterized by the two numbers $\mathrm{Y}$ (hypercharge) and I (isospin), each base vectors of the irreducible representation $\{Y, I\}$ is characterized by the 3 numbers $Y, I$ and $m_{I}$. The strong interaction is mediated through a set of 8 neutral massless vector fields, the so-called "gluons".

Let $\{3\}$ and $\left\{3^{*}\right\}$ be the two fundamental conjugated 3-dimensionals representations of $\boldsymbol{G}_{H}(6)$ or $\boldsymbol{S U}$ (3) from which all the others representations can be built [10]. The isospin doublet

$\left.Y=1 / 3, I=1 / 2, m_{I}= \pm 1 / 2\right\rangle$ and the isospin singlet $\left.Y=-2 / 3, I=0, m_{I}=0\right\rangle$ are a set of base states for $\{3\}$, the doublet $\left|Y=-1 / 3, I=1 / 2, m_{I}= \pm 1 / 2\right\rangle$ and the singlet $\left|Y=2 / 3, I=0, m_{I}=0\right\rangle$ are a set of base states for $\left\{3^{*}\right\}$. As $\boldsymbol{E}_{\perp}(6)_{M}$ has 6 dimensions, there are two such distinct triplets, the so-called quarks, plus their images by charge conjugation.

\subsection{Leptons}

Agauge field, which we call "pseudo electro-weak in- 
teraction", can be associated to the symmetry group $\boldsymbol{G}_{L}(6)$. It has the same symmetry as but it is intrinsically different from the usual electro-weak interaction. $\boldsymbol{G}_{L}(6)$ like $\boldsymbol{S U}(2) \times \boldsymbol{U}(1)$ has 4 infinitesimal generators which subtend a 4-dimension representation of $\boldsymbol{G}_{L}(6)$. Any given irreducible representation of $\boldsymbol{G}_{L}(6)$ is characterized by the two numbers $Z$ (pseudo electro-weak hypercharge) and $J$ (pseudo electro-weak isospin), the representations $\{Z, J\}$ and $\{-Z, J\}$ are conjugated, the dimension of the representation is $2 J+1$. Each of the base vectors of the irreducible representation $\{J, Z\}$ is characterized by the 3 numbers $Z, J, m_{J}$. The "pseudo electro-weak interaction" is mediated through a set of 4 neutral massless vector fields, that we call "gluinos". Those gluinos, which are bosons, shall not be confused with the so-called gluinos of the super symmetric theories, where they are the fermion partners of the gluons. The existence of this pseudo electro-weak interaction mediated by 4 extra bosons is a major difference with the Standard Model (see 4.3 hereunder).

Let $\{2\}$ and $\left\{2^{*}\right\}$ be the two fundamental conjugated 2-dimension representations of $\boldsymbol{G}_{L}(6)$ or

$\boldsymbol{S U}(2) \times \boldsymbol{U}(1)$ from which all the others representations can be built. The two states $\left|Z=1, J=1 / 2, m_{J}= \pm 1 / 2\right\rangle$ constitute a set of base states for $\{2\}$, the 2 states $\left|Z=-1, J=1 / 2, m_{J}= \pm 1 / 2\right\rangle$ constitute a set of base states for $\left\{2^{*}\right\}$. As $\boldsymbol{E}_{\perp}(6)_{M}$ has 6 dimensions, there are actually 3 distinct pairs of such doublets, plus their images by charge conjugation.

\section{ONE STEP BEYOND}

\subsection{Weak Interaction}

Since a 3-dimension hyper surface can be embedded within a 6-dimension flat space, let us consider inside $\boldsymbol{E}_{\perp}(6)_{M}$ a 3-dimension hyper surface $\boldsymbol{\Sigma}(3)_{M}$. Let $\boldsymbol{H}_{\|}(3)_{N / M}$ and $\boldsymbol{H}_{\perp}(3)_{N / M}$ respectively be the tangent space and the orthogonal space to $\boldsymbol{\Sigma}(3)_{M}$ in some point $\mathrm{N}$ of $\boldsymbol{\Sigma}(3)_{M}$. In $\boldsymbol{E}_{\perp}(6)_{M}$ the particles have 6 degrees of freedom, 3 of them in the tangent space $\boldsymbol{H}_{\|}(3)_{N / M}$ and the 3 others in the orthogonal space $\boldsymbol{H}_{\perp}(3)_{N / M}^{N / M}$. Both $\boldsymbol{H}_{\|}(3)_{N / M}$ and $\boldsymbol{H}_{\perp}(3)_{N / M}$ are 3-dimension flat spaces, invariant under the $3 \mathrm{~d}$ special orthogonal group $\boldsymbol{S O}(3)$. Actually, the choice of $\mathrm{N}$ has no effect on what follows as all the points on $\Sigma(3)_{M}$ are equivalent. The symmetry properties above only depend upon the coordinates of $\mathrm{M}$ on $\boldsymbol{\Sigma}(4)$, which is consistent with the existence of a gauge field experienced by both hadrons and leptons since we have already noticed that $\boldsymbol{G}_{H}(6)$ and $\boldsymbol{G}_{L}(6)$ have in common $\boldsymbol{S O}(3) \otimes \boldsymbol{I}(2)$ as a maximal subgroup. This gauge field is associated to the symmetry group $\mathbf{S O}(3)$, isomorphous to the special unitary symmetry $\boldsymbol{S U}(2)$, and it is identified with the weak interaction [11]. It may lift the degeneracy of the multiplets associated to the irreducible representations of $\boldsymbol{G}_{H}(6)$ and $\boldsymbol{G}_{L}(6)$. Since $\boldsymbol{S O}(3)$ has three infinitesimal generators which subtend a 3-dimensional representation $\{J=1\}$ of $\boldsymbol{S O}(3)$, the weak interaction is mediated through a triplet of neutral massless vector fields.

\subsection{Electromagnetic and BEH Interaction}

The orthogonal space $\boldsymbol{H}_{\perp}(3)_{N / M}$ to $\boldsymbol{\Sigma}(3)_{M}$ in any point $\mathrm{N}$ is also a 3-dimension space invariant under $\boldsymbol{S O}(3)$. Since a 2-dimension surface can be embedded within a 3-dimension flat space, let us consider within $\boldsymbol{\Sigma}(2)_{N / M}$ a 2-dimension hyper surface $\boldsymbol{\Sigma}(2)_{N / M}$ invariant under $\boldsymbol{S O}(3)$, i.e. $\boldsymbol{\Sigma}(2)_{N / M}$ is a sphere. Let $\boldsymbol{\Pi}(2)_{P / N / M}$ be the tangent plane to $\boldsymbol{\Sigma}(2)_{\mathrm{N} / \mathrm{M}}$ in any point $\mathrm{P}$ of $\boldsymbol{\Sigma}(2)_{N / M} \cdot \boldsymbol{\Pi}(2)_{P / N / M}$ is invariant under the 2d special orthogonal group $\boldsymbol{S O}(2)$ in any point $\mathrm{P}$ of $\boldsymbol{\Sigma}(2)_{N / M}$. Actually, the choice of P has no effect on what follows as all the points on $\boldsymbol{\Sigma}(2)_{N / M}$ are equivalent. The symmetry properties above only depend upon the coordinates of $\mathrm{M}$ on $\boldsymbol{\Sigma}(4)$, which is consistent with the existence of agauge field experienced by both hadrons and leptons and associated to the special unitary symmetry group $\boldsymbol{S O}(2)$, isomorphous to the unitary symmetry group $\boldsymbol{U}(1)$. This interaction is identified with the electromagnetic interaction. It eventually lifts the residual degeneracy of the multiplets associated to the irreducible representations of $\boldsymbol{S O}(3)$.

$\boldsymbol{S O}(2)$ has only one infinitesimal generator and its irreducible representations are of dimension 1 , each of them being characterized by a relative integer number $\mathrm{Q}$, which is identified with the electric charge. The representations $\{Q\}$ and $\{-Q\}$ are conjugated, the totally symmetric representation $\{0\}$ being self-conjugated. The interaction is mediated through a single massless vector field which is identified with the photon.

The orthogonal space to $\boldsymbol{\Sigma}(2)_{N / M}$ in $\mathrm{P}$ is a line $\Delta(1)_{P / N / M}$; in the space $\boldsymbol{H}_{\perp}(3)_{N / M}$ the particles have 3 degrees of freedom, 2 of them in the plane $\Pi(2)_{P / N / M}$, and the third one along $\Delta(1)_{P / N / M} \cdot \Delta(1)_{P / N / M}$ is a 1-dimension space whose only symmetry is $\boldsymbol{S O}(1)$ i.e. the trivial identity $\boldsymbol{I}(1)$; that can be interpreted as featuring an additional scalar field. As a consequence, both hadrons and leptons can experience a same scalar interaction mediated through a particle which can be identified with the BEH boson.

\subsection{Consequences}

To summarize, in every point $\mathrm{M}$ of the $4 \mathrm{~d}$-space-time $\boldsymbol{\Sigma}(4)$ there is a local extra 6d Euclidian space $\boldsymbol{E}_{\perp}(6)_{M}$ in which is inscribed a $3 d$ hypersphere. In any point of that hypersphere there is a 3d Euclidian subspace in which is inscribed a sphere. That unfolding, coming with a group-to-subgroup declination, reveals the interactions 
embedded as Russian dolls. The hadronic states are classified according to the symmetry

$\boldsymbol{G}_{H}(6) \times \boldsymbol{S O}(3) \times \boldsymbol{S O}(2)$ isomorphous to

$\boldsymbol{S U}(3) \times \boldsymbol{S U}(2) \times \boldsymbol{U}(1)$, and the leptonic states are classified according to the symmetry

$\boldsymbol{G}_{L}(6) \times \boldsymbol{S O}(3) \times \boldsymbol{S O}(2)$ isomorphous to

$[\boldsymbol{S U}(2) \times \boldsymbol{U}(1)] \times \boldsymbol{S U}(2) \times \boldsymbol{U}(1)$.

As $\boldsymbol{S O}(2)$ and $\boldsymbol{I}(1)$ are orthogonal subgroups of $\boldsymbol{S O}(3)$, the BEH boson has no electric charge and the photon has no mass. Due to the effect of the electromagnetic field on the one hand, and of the BEH field on the other hand, the fundamental representation $\{J=1\}$ of $\boldsymbol{S O}(3)$ is splitted into a singlet (charge $Q=0$, mass $m=m^{0}$ ) and a doublet (charge $Q= \pm 1$, masse $m=m^{0}+\delta m$ ) so that the 3 mediators of the weak interaction above gain an electric charge and a mass; they are respectively identified with the $Z^{0}$ and $W^{ \pm}$particles. The same mechanism confers an electric charge and a mass to hadrons and leptons.

We have thus evidenced 3 interactions common to both hadrons and leptons and which can be identified with the weak, elecromagnetic and BEH interactions. Finally we have one scalar coupling and a set of fields respectively mediated through 8 gluons, 4 gluinos, 3 bosons $\left(W^{+}, W^{-}, Z^{0}\right), 1$ photon. The vector character of the gluons, gluinos, $W^{+}, W^{-}, Z^{0}$ and photon is verified in the Annex [12].

The hypothetical existence of a pseudo electro-weak interaction mediated by the 4gluinos is a major difference with the Standard Model based upon the

$\boldsymbol{S U}(3) \times \boldsymbol{S U}(2) \times \boldsymbol{U}(1)$ gauge symmetry. It has been shown [13] that a Kaluza-Klein-type theory consistent with the Standard Model requires 11 dimensions, with 7 additional compactified dimensions. Now, that pseudo electro-weak interaction superposed to the usual electro-weak interaction which has the same symmetry is presumably very weak.

If the "orbital" degrees of freedom are taken into account, the resulting states of fields and particles will be classified according to the irreducible representations of $\boldsymbol{\Lambda}(4) \times \boldsymbol{G}_{H}$ (6) for hadrons and $\boldsymbol{\Lambda}(4) \times \boldsymbol{G}_{L}$ (6) for leptons, built from the irreducible representations of $\Lambda(4)$ and $\boldsymbol{G}_{H}(6)$ or $\boldsymbol{G}_{L}(6)$.

We notice that inside the "internal space" $\boldsymbol{E}_{\perp}(6)_{M}$ the charge conjugation simply appears as the equivalent of what are parity and time reversal in the "orbital space" $\boldsymbol{E}_{\|}(4)_{M}$, i.e. the transformation $\xi^{i} \rightarrow-\xi^{i}$ within ranging from 1 to 6 is analogous to $x^{\mu} \rightarrow-x^{\mu}$ with $\mu$ ranging from 0 to 3 .

\section{THE GRAVITATION FIELD}

We now consider again the original 10-dimensional space $\boldsymbol{E}(10)$, which has been assumed to be globally invariant under a symmetry group $\mathcal{G}(10)$. In any point
M of the 4-dimension surface $\boldsymbol{\Sigma}(4) \boldsymbol{E}(10)$ is the tensor product of a 4-dimensional "orbital” space invariant under the Lorentz group $\boldsymbol{\Lambda}(4)$ and of a 6-dimension "internal” space $\boldsymbol{E}_{\perp}(6)_{M}$ invariant under $\boldsymbol{S O}(6)$. $\boldsymbol{S O}(6)$ and $\boldsymbol{\Lambda}(4)$ are both invariant, or distinguished, subgroups of $\mathcal{G}(10)$.

In the previous sections, we have considered several gauge fields associated to symmetries of the "internal" space. Now gravitation can also be considered as a gauge field by which the global Lorentz invariance of special relativity is changed into a local invariance at any point M of $\Sigma(4)$.

With 4 dimensions, if the global Lorentz invariance of special relativity is changed into a local symmetry on the 4-dimension hyper surface, then $\partial_{\mu}=\frac{\partial}{\partial x^{\mu}}$ is changed into

$$
D_{\mu}=\partial_{\mu}+\boldsymbol{G}_{\mu}^{v} \partial_{v}
$$

with $\mu$ ranging from 0 to 3, where $\boldsymbol{G}_{\mu}^{v}$ is a tensor quantity featuring the local geometry of $\Sigma(4)$. The impulsion

$$
\boldsymbol{p}^{\mu}=\mathrm{i}^{-1} \partial^{\mu}=\mathrm{i}^{-1} \boldsymbol{\eta}^{\mu v} \partial_{v}=\left(\mathrm{i} \frac{\partial}{\partial t}, \mathrm{i}^{-1} \nabla\right)
$$

is thus changed into

$$
\boldsymbol{P}^{\mu}=\mathrm{i}^{-1} D^{\mu}=\boldsymbol{p}^{\mu}+\boldsymbol{G}_{v}^{\mu} \boldsymbol{p}^{v} .
$$

There is some flexibility in the determination of the $\boldsymbol{G}_{\mu}^{v}$ 's which allows to impose the 4 conditions

$$
\partial_{\mu} \boldsymbol{G}_{v}^{\mu}=0 \text {. }
$$

We will now see how this can be extended if the 4dimension surface is supposed to be embedded in the full 10-dimension flat space-time. In the previous sections, we had separately assumed the conservation of the pseudo Euclidian norm $\boldsymbol{\eta}_{\mu v} x^{\mu} x^{v}=-c^{2} t^{2}+x^{2}+y^{2}+z^{2}$ within $\boldsymbol{E}_{\|}(4)_{M}$ and the conservation of a true Euclidian norm $\sum_{i=1}^{6}\left(\xi^{i}\right)^{2}$ within $\boldsymbol{E}_{\perp}(6)_{M}$. We now further postulate the conservation of the full pseudo Euclidian norm $-c^{2} t^{2}+x^{2}+y^{2}+z^{2}+\sum_{i=1}^{6}\left(\xi^{i}\right)^{2}$ within $\boldsymbol{E}(10)$. The full 10-dimension space $\boldsymbol{E}(10)$ is thus assumed to be a pseudo Euclidian space which preserves a pseudo norm $\boldsymbol{\eta}_{\alpha \beta} x^{\alpha} x^{\beta}$ with $\alpha$ and $\beta$ ranging from 0 to 9, i.e. $\boldsymbol{\eta}_{00}=-1, \boldsymbol{\eta}_{\alpha \alpha}=1$ if $\alpha \neq 0, \boldsymbol{\eta}_{\alpha \beta}=0$ otherwise.

Let us postulate that in a 10-dimension reference frame of $\boldsymbol{E}(10)$, the Lagrangian $\mathfrak{L}$ attached to a field $\Theta\left(x^{\alpha}\right)$ simply is

$$
\mathfrak{L}=\boldsymbol{\eta}^{\alpha \beta} \dot{\Theta}_{\alpha}^{\dagger} \dot{\Theta}_{\beta} \text {, with } \dot{\Theta}_{\alpha}=\partial_{\alpha} \Theta
$$


Now taking into account the local character of the symmetries with respect to the reference frame $\left\{c t, x, y, z, \xi^{1}, \xi^{2}, \xi^{3}, \xi^{4}, \xi^{5}, \xi^{6}\right\}$ attached to the point $\mathrm{M}$ of $\boldsymbol{\Sigma}(4)$, we extend to $\boldsymbol{E}(10)$ what has been done above with the Lorentz invariance in the case of a 4-dimension space-time. The transformation Equation (7) readily becomes

$$
\partial_{\alpha} \rightarrow D_{\alpha}=\partial_{\alpha}+\boldsymbol{G}_{\alpha}^{\beta} \partial_{\beta}
$$

And the Lagrangian Equation (11) is changed into

$$
\mathfrak{L}=\boldsymbol{\eta}_{\alpha \beta}\left[\boldsymbol{P}^{\alpha} \Theta\right]^{\dagger}\left[\boldsymbol{P}^{\beta} \Theta\right]
$$

or

$$
\mathfrak{L}=\boldsymbol{g}_{\alpha \beta}\left[\boldsymbol{p}^{\alpha} \Theta\right]^{\dagger}\left[\boldsymbol{p}^{\beta} \Theta\right]
$$

where we have introduced the effective $10 \times 10$ metric tensor

$$
\boldsymbol{g}_{\alpha \beta}=\boldsymbol{\eta}_{\alpha \beta}+\boldsymbol{h}_{\alpha \beta}
$$

with

$$
\boldsymbol{h}_{\alpha \beta}=\boldsymbol{\eta}_{\rho \sigma} \boldsymbol{G}_{\alpha}^{\rho} \boldsymbol{G}_{\beta}^{\sigma}+\boldsymbol{\eta}_{\alpha \rho} \boldsymbol{G}_{\beta}^{\rho}+\boldsymbol{\eta}_{\beta \sigma} \boldsymbol{G}_{\alpha}^{\sigma}
$$

From the expression of $\boldsymbol{g}^{\alpha \beta}$ as a function of the $\boldsymbol{G}_{\alpha \beta}$ $\mathrm{s}$ it is obvious that $\boldsymbol{g}^{\alpha \beta}=\boldsymbol{g}^{\beta \alpha}$. Applying the Lagrange equations to the $\Theta$ field, i.e.

$$
\partial_{\alpha} \frac{\partial \mathfrak{L}}{\partial \dot{\Theta}_{\alpha}}=\frac{\partial \mathfrak{L}}{\partial \Theta},
$$

gives

$$
\boldsymbol{g}^{\alpha \beta} \partial_{\alpha} \partial_{\beta} \Theta+\left(\partial_{\alpha} \boldsymbol{g}^{\alpha \beta}\right)\left(\partial_{\beta} \Theta\right)=0
$$

The gauge properties give some flexibility in the determination of the $\boldsymbol{G}_{\beta}^{\alpha}$ 's so that we can impose the 10 conditions

$$
\partial_{\alpha} \boldsymbol{G}_{\beta}^{\alpha}=0
$$

from which we derive

$$
\partial_{\alpha} \boldsymbol{g}^{\alpha \beta}=0
$$

The equation of evolution Equation (18) thus becomes

$$
\boldsymbol{g}^{\alpha \beta} \partial_{\alpha} \partial_{\beta} \Theta=0
$$

That expression means that the only accessible physical states are those whose measure is null in $\boldsymbol{E}(10)$. With the correspondence $\boldsymbol{p}^{\alpha} \leftrightarrow \mathrm{i}^{-1} \hbar \partial^{\alpha}$, one equivalently gets

$$
\boldsymbol{g}_{\alpha \beta} \boldsymbol{p}^{\alpha} \boldsymbol{p}^{\beta} \Theta=0 .
$$

$\boldsymbol{g}$ has the following form

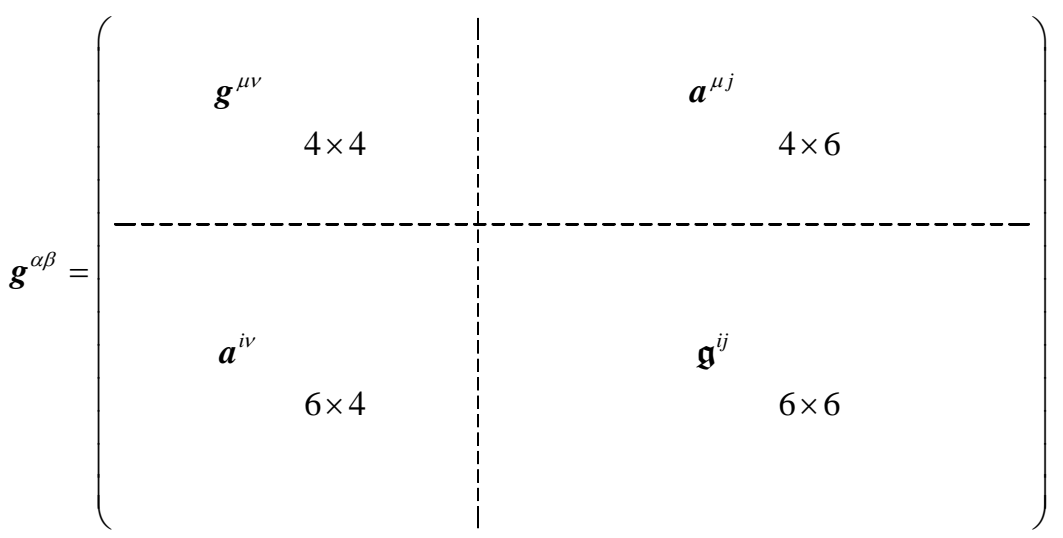

Since $\boldsymbol{g}^{\alpha \beta}=\boldsymbol{g}^{\beta \alpha}$ and $\partial_{\alpha} \boldsymbol{g}^{\alpha \beta}=0, \boldsymbol{g}$ has 55 distinct components, of which 45 are independent.We can also choose $\partial_{\mu} \boldsymbol{g}^{\mu v}=0$, with $\mu$ and $v$ ranging from 0 to 3 , and $\partial_{i} \mathfrak{g}^{i j}=0$, with $i$ and $j$ ranging from 4 to 9 , so that $\boldsymbol{g}^{\mu \nu}, \mathfrak{g}^{i j}$ and $\boldsymbol{a}^{i v}$ respectively have 10, 21 and 24 distinct components, of which 6,15 and 24 are independent.
Now, we look for $\Theta$ special solutions which can be expressed as a product

$$
\Theta\left(c t, x, y, z, \xi^{1}, \cdots, \xi^{6}\right)=\Psi(c t, x, y, z) \Phi\left(\xi^{1}, \cdots, \xi^{6}\right),(24)
$$

With the expressions Equation (23) for $\boldsymbol{g}$ and Equation (24) for $\Theta$, the equation of evolution becomes

$$
\left[\boldsymbol{g}^{\mu v} \partial_{\mu} \partial_{v}+\mathfrak{g}^{i j} \partial_{i} \partial_{j}+\boldsymbol{a}^{\mu j} \partial_{\mu} \partial_{j}\right] \Psi(c t, x, y, z) \Phi\left(\xi^{1}, \cdots, \xi^{6}\right)=0
$$

Multiplying Equation (25) by $\Phi^{\dagger}$ and integrating over the 6 degrees of freedom of $\boldsymbol{E}_{\perp}(6)_{M}$ yields $\boldsymbol{g}^{\mu \nu} \partial_{\mu} \partial_{v} \Psi=-\left\langle\Phi\left|\mathfrak{g}^{i j} \partial_{i} \partial_{j}\right| \Phi\right\rangle \Psi-\left\langle\Phi\left|\boldsymbol{a}^{\mu j} \partial_{j}\right| \Phi\right\rangle \partial_{\mu} \Psi(26)$ 
with $\dot{\Psi}_{\mu}=\partial_{\mu} \Psi$, or

$$
\boldsymbol{g}^{\mu v} \partial_{\mu} \partial_{\nu} \Psi=\hbar^{-2}\left\langle\Phi\left|\mathfrak{g}_{i j} \boldsymbol{p}^{i} \boldsymbol{p}^{j}\right| \Phi\right\rangle \Psi-\left\langle\Phi\left|\boldsymbol{a}^{\mu j} \partial_{j}\right| \Phi\right\rangle \dot{\Psi}_{\mu}
$$

i.e.

$$
\boldsymbol{g}^{\mu v} \partial_{\mu} \partial_{v} \Psi=\hbar^{-2}\left\langle\mathfrak{g}_{i j} \boldsymbol{p}^{i} \boldsymbol{p}^{j}\right\rangle \Psi-\left\langle\Phi\left|\boldsymbol{a}^{\mu j} \partial_{j}\right| \Phi\right\rangle \dot{\Psi}_{\mu} .
$$

Let us consider the first term on the right hand side of the above equation and in first approximation let us neglect the second term. We get

$$
\boldsymbol{g}^{\mu v} \partial_{\mu} \partial_{v} \Psi=\hbar^{-2}\left\langle\boldsymbol{g}_{i j} \boldsymbol{p}^{i} \boldsymbol{p}^{j}\right\rangle \Psi
$$

It can be compared with the Klein-Gordon equation in the presence of a gravitation field

$$
\boldsymbol{g}^{\mu v} \partial_{\mu} \partial_{v} \Psi(c t, x, y, z)=(m c / \hbar)^{2} \Psi(c t, x, y, z)
$$

where $m$ is the particle mass. Identifying $m$ with the self energy term in Equation (29), i.e.

$$
m c=\left\langle\mathfrak{g}_{i j} \boldsymbol{p}^{i} \boldsymbol{p}^{j}\right\rangle^{1 / 2},
$$

means that the particle mass originates from the 6 extra dimensions of space-time, i.e. from the symmetries of the local orthogonal space $\boldsymbol{E}_{\perp}(6)_{M}$.

Now let us focus on the second term on the right hand side of Equation (28). It can be interpreted as the coupling between $\Psi$ and a set of 6 additional massless vector fields

$$
\boldsymbol{A}^{\mu}=\left\langle\Phi\left|\boldsymbol{a}^{\mu j} \partial_{j}\right| \Phi\right\rangle
$$

so that the field equation for $\Psi$ can be written as

$$
\boldsymbol{g}^{\mu v} \partial_{\mu} \partial_{\nu} \Psi=(m c / \hbar)^{2} \Psi-\boldsymbol{A}^{\mu} \dot{\Psi}_{\mu} .
$$

Those extra fields express the connection between gravitation in the "orbital" 4-dimension space-time and the other interactions which have their origin in the symmetry properties of the "internal" space $\boldsymbol{E}_{\perp}(6)_{M}$.

A priori those fields are massless and thus they have an infinite range; they are superposed to gravitation, but should be much weaker so as to be in accordance with the experimental data. Now if they get a mass through some mechanism, e.g. via an additional scalar field, they then may have a finite range and consequently the laws of gravitation should be modified below some scale. That could account for the dark matter/dark energy problem.

The hypothetical existence of those vector companions of gravity implies a violation of the equivalence principle. The way the gravitation field has been here above introduced in the 4-dimension space-time naturally implies the equivalence between gravitational mass and inertial mass. However if we consider the full $\boldsymbol{E}(10)$ space we have seen that the hadrons experience a symmetry $\boldsymbol{G}_{H}(6)$ isomorphous to $\boldsymbol{S U}(3)$ whereas the leptons experience a symmetry $\boldsymbol{G}_{L}(6)$ isomorphous to
$\boldsymbol{S U}(2) \times \boldsymbol{U}(1)$. As a consequence, the interactions $\boldsymbol{A}^{\mu}$ should be different for hadrons and leptons and thus bodies with different composition would not behave the same way under $\boldsymbol{A}^{\mu}$. Experimentally that would result in a violation of the equivalence principle [14].

\section{CONCLUSION}

We have given a coherent presentation of the fundamental interactions of physics including gravitation by considering the symmetry properties of a 10-dimension real space-time. The usual space-time is a 4-dimensional surface $\boldsymbol{\Sigma}(4)$, whose local symmetry is the Lorentz group $\boldsymbol{\Lambda}(4)$, embedded in a flat 10-dimensional space $\boldsymbol{E}(10)$. In every point of that "orbital" space $\boldsymbol{\Sigma}(4)$, there is a 6-dimension "internal" space $\boldsymbol{E}_{\perp}(6)_{M}$, orthogonal to the surface $\Sigma(4)$. Simple assumptions about the geometry of $\boldsymbol{E}_{\perp}(6)_{M}$ : allow deriving the following: hadrons and leptons, 8 gluons for the strong interaction, 4 gluinos for a pseudo electro-weak interaction, 3 intermediate bosons for the weak interaction, 1 photon, plus an additional scalar field identified with the BEH field.

When gravitation is introduced, the connection between the "orbital" and the "internal" degrees of freedom shows that mass has its origin in the "internal" space and that there exists additional couplings, mediated by a set of 6 extra vector fields. Those hypothetical companions of gravity do not act the same way with hadrons and leptons and should be revealed through a violation of the equivalence principle.

\section{REFERENCES}

[1] Isham, C. (1995) Structural issues in quantum gravity.

[2] Fayet, P. (1996) The standard model and beyond. History of Original Ideas and Basic Discoveries in Particle Physics, Proceedings of Erice Conference, Plenum Press, New York.

[3] Randall, L. and Sundrum, R. (1999) Large mass hierarchy from a small extra dimension. Physical Review Letters, 83, 3370. http://dx.doi.org/10.1103/PhysRevLett.83.3370

[4] Raby, S. (2004) Grand unified theories. In: Eidelman, S. et al., Ed., The Review of Particle Physics, Physical Letters $B, 592,1$. http://dx.doi.org/10.1016/j.physletb.2004.06.001

[5] Giudice, G.F. and Wells, J.D. (2006) Extra dimensions. In: Yao, W.-M., et al., Review of Particle Physics, 33, 1. http://dx.doi.org/10.1088/0954-3899/33/1/001

[6] Marolf, D. (2004) Resource letter NSST-1: The nature and status of string theory.

[7] Dupays, A., Lamine, B. and Blanchard, A. (2013) A\&A, A60, 554.

[8] Berestetski, V., Lifchitz, E. and Pitayevski, L. (1967) Relativistic quantum theory, Part 1, MIR ed., Moscow.

[9] Hammermesh, M. (1964) Group theory. Addison-Wesley. 
[10] Lifchitz, E. and Pitayevski, L. (1967) Relativistic quantum theory, Part 2, MIR ed., Moscow.

[11] T’Hooft, G. and Veltman, M.J.G. (2000) A confrontation with infinity: From weak interactions to gravitation. Review of Modern Physics, 72, 333-349.

[12] Weinberg, G. (1974) Recent progress in gauge theories of the weak, electromagnetic and strong interactions. Review of Modern Physics, 46, 255-277.

\section{ANNEX}

In the main section, w e have evidenced one scalar interaction (identified with the $\mathrm{BEH}$ field) and a set of fields respectively mediated through 8 gluons, 4 gluinos, 3 bosons identified with the $W^{+}, W^{-}, Z^{0}$ and 1 photon, each of them being associated to a symmetry group $\boldsymbol{G}$. The elementary operations associated to any of them have the form

$$
\boldsymbol{T}=\boldsymbol{I}+\mathrm{i} \sum_{p} \boldsymbol{N}_{p}
$$

where $\boldsymbol{N}_{p}$ is an infinitesimal generator of $\boldsymbol{G}$. More generally, any operation of $\boldsymbol{G}$ can be written as

$$
\boldsymbol{T}=\operatorname{expi} \sum_{p} \boldsymbol{N}_{p}
$$

We now consider a scalar particle of mass $m$ (but the procedure can be generalized to any spin) associated to the field $\Psi(c t, x, y, z)$ in the 4-dimension space-time. Its Lagrangian density is

$$
\mathfrak{L}=\boldsymbol{\eta}^{\mu v} \dot{\Psi}_{\mu}{ }^{\dagger} \dot{\Psi}_{v}+(m c / \hbar)^{2} \Psi^{\dagger} \Psi \text {, with } \dot{\Psi}_{\mu}=\partial_{\mu} \Psi
$$

or

$$
\mathfrak{L}=\boldsymbol{\eta}_{\mu v} \hbar^{-2}\left[\boldsymbol{p}^{\mu} \Psi\right]^{\dagger}\left[\boldsymbol{p}^{v} \Psi\right]+(m c / \hbar)^{2} \Psi^{\dagger} \Psi
$$

\section{http://dx.doi.org/10.1103/RevModPhys.46.255}

[13] Witten, E. (1981) Search for a realistic Kaluza-Klein theory. Nuclear Physics, B186, 412. http://dx.doi.org/10.1016/0550-3213(81)90021-3

[14] Damour, T. (1996) Testing the equivalence principle: Why and how? Proceedings of Fundamental Physics in Space Symposium (London), Classics of Quantum Gravity, 13, 33-41.

with $\mu$ and $v$ ranging from 0 to 3 .

If a global symmetry $\boldsymbol{T}=\operatorname{expi} \sum_{p} \boldsymbol{N}_{p}$ is assumed to be a local one, i.e. $N_{p}=N_{p}\left(x^{\mu}\right)$, then

$$
\begin{aligned}
& \boldsymbol{p}^{\mu} \Psi \rightarrow \boldsymbol{p}^{\mu}\left(\operatorname{expi} \sum_{p} \boldsymbol{N}_{p}\right) \Psi \\
& =\left(\operatorname{expi} \sum_{p} \boldsymbol{N}_{p}\right)\left(\boldsymbol{p}^{\mu} \Psi+\hbar \sum_{p} \partial^{\mu} \boldsymbol{N}_{p} \Psi\right)
\end{aligned}
$$

Writing the full state of the particle as a product of the "orbital" state $\Psi(c t, x, y, z)$ times an "internal" state $\Phi\left(\xi^{1}, \cdots, \xi^{6}\right)$, the conservation of the Lagrangian imposes inside $\Sigma(4)$ the existence of a vector-type gauge field

$$
\boldsymbol{A}^{\mu}=\sum_{p} \partial^{\mu}\left\langle\Phi\left|\boldsymbol{N}_{p}\left(x^{\mu}\right)\right| \Phi\right\rangle
$$

after averaging over the 6 degrees of freedom $\left\{\xi^{i}\right\}$ since $\boldsymbol{N}_{p}$ exclusively acts upon the variables $\left\{\xi^{i}\right\}$. It implies the vector character of the above fields: gluons, gluinos, $W^{+}, W^{-}, Z^{0}$ and photon. 